\title{
Design and Analysis of Resonant Wireless Power Transfer System
}

\author{
Masood Rehman ${ }^{1,}{ }^{*}$, Zuhairi Baharudin ${ }^{1, \dagger}$, Perumal Nallagownden ${ }^{1}$, Badar ul Islam ${ }^{1}$ \\ ${ }^{1}$ Electrical and Electronics Engineering Department, Universiti Teknologi PETRONAS, 32610 Seri \\ Iskandar, Perak, Malaysia.
}

\begin{abstract}
In this paper, the modelling and analysis of resonant wireless power transfer (RWPT) system are carried out by using circuit theory. Equations of the efficiency and frequency are derived for basic series-series circuit topology and series-parallel-mixed circuit topology. The designed model uses the series-parallel-mixed topology. The model is tested for two different peak-to-peak voltage levels including $20 \mathrm{~V}$ and $40 \mathrm{~V}$. The achieved efficiency at $20 \mathrm{~V}$ is $49.10 \%$ and at $40 \mathrm{~V}$ is $49.52 \%$ approximately. The modelling is performed by using advanced design system (ADS) high frequency design software. In addition, an overview of currently available wireless power transfer (WPT) technologies and the recent study on inductive wireless power transfer (IWPT) and resonant wireless power transfer (RWPT) systems is also presented.
\end{abstract}

\section{Introduction}

Wireless power transfer (WPT) was first reported in the early 20th century and the idea was given by Nikola Tesla [1]. According to power transfer technique, WPT can be radiative or non-radiative. Radiative power depends on far field. The power can be transferred through a medium such as vacuum or air via an antenna over long distance in form of an electromagnetic wave [2]. The non-radiative wireless power transfer uses near-field inductive coupling or resonant coupling. It is suitable for short-range and mid-range applications. The radiative and non-radiative power transfer can be further classified as illustrated in Fig. 1. Recent research in WPT has highlighted the potential benefits of transferring the wirelessly by non-radiative mode. The non-radiative power transfer mechanism includes inductive wireless power transfer (IWPT) and resonant wireless power transfer (RWPT).

The IWPT mechanism uses electromagnetic induction technique for transferring the electrical energy wirelessly from primary to secondary coil. IWPT's working principle is almost like that of basic transfer. In a transformer, the magnetic field is typically restricted to high permeability core. But on the other hand, in the IWPT technique the separation area between the primary and secondary loops can be simply air [3]. The RWPT is the reformed and improved form of the IWPT technology. It takes the benefit of resonance state of coils and uses the resonance coupling to enhance the efficiency by getting the maximum amount

\footnotetext{
* Corresponding author: masood.rehman@ hotmail.com

$\dagger$ Corresponding author: zuhairb@utp.edu.my
} 
of power at the receiver side. Resonance coupling can be created by using self-resonance of the helix coils, which resonates at a certain frequency by using its self-inductance and its parasitic capacitance. But when the parasitic capacitance of the resonating coils are inadequate to achieve a resonance at the required frequency, an external capacitor need to be added in order to realize the resonance state of coils [4].

Fig. 1. Categorization of WPT technology

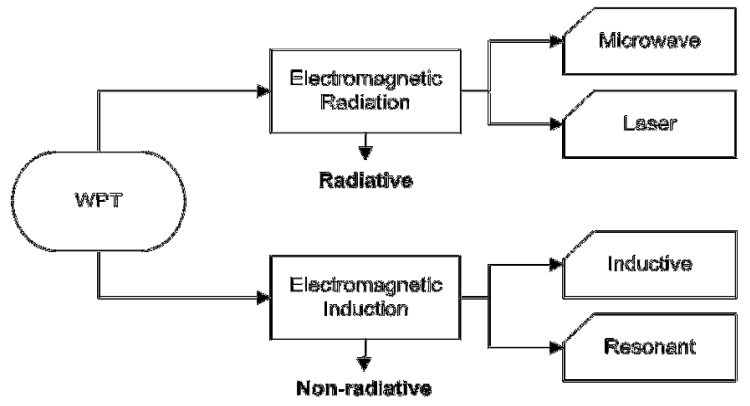

\subsection{Overview of IWPT and RWPT techniques}

IWPT is the promising near field simple technology having short-transmission distances up to few centimetres. Although it offers higher power transfer efficiency, however it needs accurate alignment for the charging path between transmitter and receiver. Charging pads for cell phones, laptops, electrical vehicle charging and chargers for electrical toothbrushes are the examples of IWPT technology. It works between the frequencies of several $\mathrm{kHz}$ to a few $\mathrm{MHz}$ [5]. RWPT is also a near field technology and appropriate for mid-range applications from few centimetres to few meters. In the RWPT technique, the leakage inductance is compensated by utilizing the techniques of magnetic coupling and resonance together for achieving the improved power transfer efficiency. In RWPT technology, the effective power transfer efficiency (PTE) over few meters distance can be achieved, but the switching frequency of RWPT is often much higher (i.e. from few $\mathrm{MHz}$ up to several $\mathrm{MHz}$ ) as compare to IWPT [6].

The research published in [7] provides a comparative analysis between IWPT and RWPT. The Authors have designed a WPT model for charging the small battery of implantable devices. They studied the behaviour of the model and analysed that it works like the traditional IWPT when the operating frequency is not resonant. However, by adjusting the resonant operating frequency, the developed model works like RWPT. In addition, the finite element analysis method was used for quantitative comparative analysis of that WPT model. The Analysis uses diverse frequency ranges from $0.01 \mathrm{MHz}$ to $10 \mathrm{MHz}$. The prototype of the model was also fabricated to verify and validate the simulation results. From the results, it has been verified that the efficiency of RWPT is much higher than that of traditional IWPT system for mid-range distances. Furthermore, the research reported in [8] proved that there is a "magic regime" in magnetically coupled resonators, where efficiency remains almost constant for a certain distance. This regime is not available in conventional IWPT system.

In [9], Authors have proposed an optimizable WPT system for obtaining higher power transfer efficiency. They were able to achieve an efficiency of $45 \%$ at $20 \mathrm{~cm}$ and an increase in efficiency up to $85 \%$ was realized at comparatively less distance of $10 \mathrm{~cm}$. In addition, their proposed model is suitable for further optimization. The model can be optimized by varying the circuit parameters at different ranges between the transmitter and receiver coils for achieving better results. This paper [10] presents a method, which includes three steps: 1) system calibration, 2) coarse search and 3) fine search. In this paper, a robust impedance 
matching technique is proposed and the WPT system is analysed for several misalignments between transmitter and receiver with distance variations. After the experiments, it is observed that the proposed technique significantly reduces the searching time from a few minutes to about one second by robust impedance searching capability and provides good accuracy of impedance matching. That leads to the enhancement of efficiency. At $6.78 \mathrm{MHz}$, the power transmission efficiency of more than $80 \%$ up to distance 50 centimetres is obtained. For centre-to-centre misalignments of around 35 centimetres, the power transfer efficiency is significantly enhanced up to $74.1 \%$, which was $48.4 \%$ without impedance matching.

The focus of this study is on RWPT technology, because it offers better efficiency as compare to IWPT at mid-range distances. Although the lot of work has been done for the implementation of RWPT technology but still this technology is ineffective because of the following constraints. The power transfer efficiency of RWPT decreases rapidly when the distance between transmitter and receiver increases. Therefore, it is essential to optimize the design of currently available resonators to improve the efficiency of RWPT. Additionally, the decrease of resonant frequency is required, because the presently available power electronics devices are ineffective and difficult to implement for generating high frequency $\mathrm{AC}$ supply and they produces losses in high frequency AC supplies. In order to reduce these losses, the frequency decrease analysis should be carried out to study the effects of decreasing the operating frequency on overall system efficiency without changing resonant condition. The parameters including inductance, capacitance, resistance, voltage source and resonance frequency plays an important role in the design of WPT system. Therefore, an investigation can be carried out by varying the above parameters. Furthermore, the analysis can be also done by varying the distance between the transmitter and the receiver.

The purpose of this research is to design the RWPT model for achieving better efficiency. The designed model uses the parallel-series-mixed topology. Analysis of the model is carried out by using circuit theory. The model is tested for different levels of supply voltages to investigate the over-all performance of the model. This paper is organized in such a manner that the section 2 includes the literature survey of WPT technology. Section 3 of this paper consists of the modelling of resonant wireless power transfer system. Simulation results are given in the section 4 and conclusion is presented in section 5 .

\section{Literature Review}

Nikola Tesla has performed several experiments about a century ago for wireless power transfer. Although his idea was good, but could not get enough attention, because the radio technology was not that advanced in those days. Fig. 2 shows the experimental setup performed by Tesla in 1914 [11].

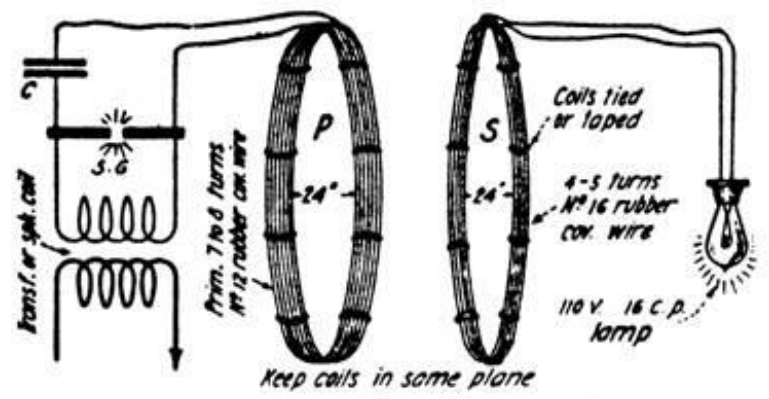

Fig. 2. Tesla Apparatus and Experiments by H. Winfield Sector, Practical Electrics [11] 
Moreover, Tesla tried to implement his plan for supply the wireless electrical energy at a very far distances. For this purpose, the famous Tesla Wardenclyffe Tower was erected in 1901. However that project was not successful and the tower was demolished in 1917 [12]. After the Tesla's experiments, there has been very little research on wireless power transfer technology. This technology was able to get the world's attention again, when the MIT researcher's team introduced a magnetic resonant coupled scheme to increase the transmission efficiency for mid-range distances $[13,14]$. As shown in Fig. 3, they utilized four coils, which consist of sending coil, source coil, receiving coil and load coil. They used the source and the load coils with much fewer turns than the sending and the receiving coils. In the experiment, they succeeded to light the $60 \mathrm{~W}$ bulb at $2 \mathrm{~m}$ air gap using two self-resonant spiral coils of radius $30 \mathrm{~cm}$ and they achieved the transfer efficiency of about $40 \%$ to $60 \%$ $[14,15]$.

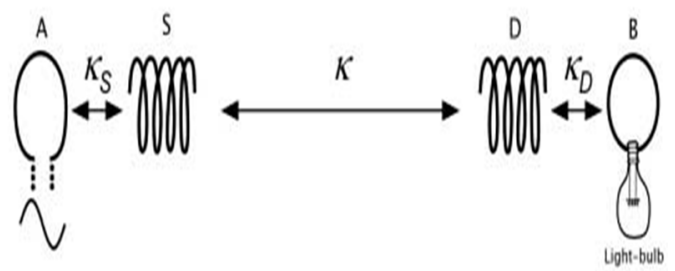

Fig. 3. Schematic diagram of the experimental setup by MIT [14]

After the development of inductive and resonant wireless power technologies, the cordless charging of portable low power electronic devices was initially implemented and commercialized for tooth brushes, cell phones [16] and for other similar low power electronic devices. Thereafter, the WPT technique has also been implemented for high power applications in kilowatts (KW) range, such as charging of electric vehicles [17]. It is worthwhile to mention that the market of wireless charging has been grown rapidly after the universal standardization of wireless charging. In the year 2015, more than 140 million of wireless charging receivers have been sold and more than 50 million wireless technology chargers have been sold. It is predicted that this number is going to be double in near future [18].

Nowadays, many researchers in industry and academia are working on non-radiative WPT technology. Their focus is on the improvement of power transfer efficiency (PTE) and enhancement of air gap between the transmitter (Tx) and receiver (Rx). In addition, the misalignment between the coils is also the topic of interest for the researchers. As presented in [19-21], the misalignment between $\mathrm{Tx}$ and $\mathrm{Rx}$ can significantly deteriorate the efficiency of wireless power transfer. Numerous interesting works have been done so far with different kinds of innovative circuits, as well as the system analysis and control in [22-24]. The detailed discussion regarding multi-dimensional WPT structure is reported in [25]. Furthermore, the frequency splitting phenomenon is discussed in the literature. This phenomenon takes place in the WPT systems with multiple transmitter and receiver coupling, when two or more adjacent coils are very close to each other in a way that they share strong relative magnetic fields. It is analysed in detail by using circuit theory in [26]. The resonance frequency of $10 \mathrm{MHz}$ is used for the simulations. It is examined that when the coupling coefficient is sufficiently large, two splitting frequencies arises, i.e. $9.5 \mathrm{MHz}$ and $10.5 \mathrm{MHz}$. The selection of better frequency adjustment for achieving maximum power transfer is done by simulation of the magnetic field distribution. In [27], a novel model of two strongly coupled resonators including a transmitter and a receiver is proposed as illustrated in Fig. 4. Transmitter consists of $L_{T X}, L_{B S T, T X}$ and a receiver also includes two coils $L_{B S T, R X}$ and $L_{R X}$. It is different from four coil conventional model in a way that, the first and fourth coil also participate in strong cross coupling with high loaded quality factor $(Q)$. With experiments, it is proved that the proposed model gives $65.2 \%$ efficiency with $17.2 \mathrm{~W}$ power transfer at 
$13 \mathrm{~cm}$ distance, whereas those values with conventional four coil technique are only $37.3 \%$ and $6.2 \mathrm{~W}$ at the same distance. Note that the IRF530NPbF is used as the switch MOSFET in this model. Further, the rectification stage of the series-resonant Rx contains a full bridge rectifier and a filtering capacitor. For the parallel-resonant Rx, an additional DC inductor is inserted at the filtering stage.

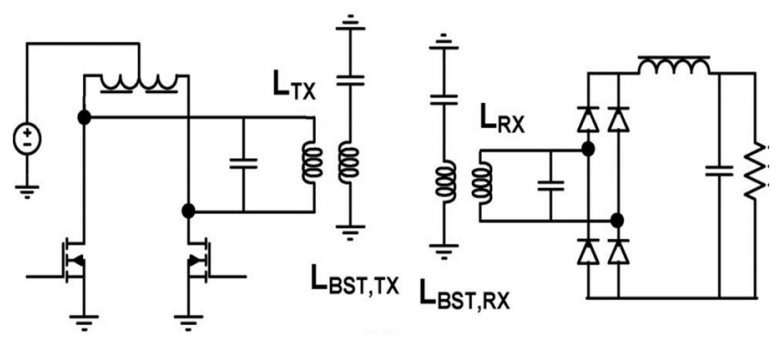

Fig. 4. Schematic diagram of four coil resonators using MOSFET [27]

The research presented in [28] the authors have proposed an innovative $5 \mathrm{~m}$ off distance wireless power transfer circuit with optimized design of stepped core as shown in Fig. 5. The operation of the circuit is performed by using $20 \mathrm{kHz}$ switching frequency. The circuit with optimized core verified to have only $41 \%$ core loss as compared with the typical even core but supplies 2.1 times more power for a specified size of core. It is found that at the various distances of 3, 4 and 5 meters, the obtained efficiencies are $29 \%, 16 \%, 8 \%$ with power outputs of 1403, 471, 209 Watts respectively.

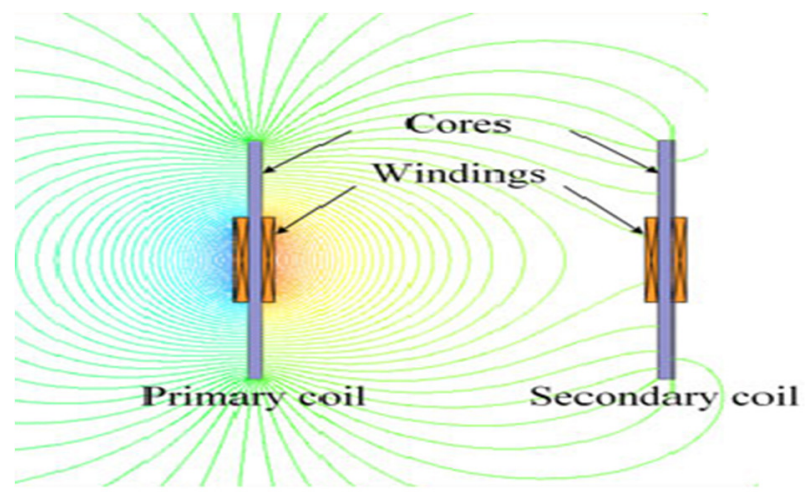

Fig. 5. Simulation result of the magnetic flux lines of the proposed Inductive Power Transfer System in [28]

Furthermore, in [29], It was concluded that the maximum distance between Tx and Rx is associated with the radius and number of turns of the coils. The research regarding the IWPT technique is reported in $[17,30]$, which provides the implementation of IWPT technique for high power applications. Currently, at kilowatts power level, a power transfer efficiency above $90 \%$ is obtainable for short distance applications [31]. In the research [32], the authors have proposed a 4 coil structure with transmitting and receiving coils having high quality factors. The proposed model uses very high frequency of $27.50 \mathrm{MHz}$ and it was discovered that the model was improved compare to other related models with promising operating characteristics over longer distances, even when smaller coils are used. But the reason can be the very high efficiency, which is very difficult to implement and can be harmful for human health. The maximum distances measured in the experiment was $4 \mathrm{~m}$. The research in [33] presented the critical review on RWPT. The review concluded that high frequencies and highquality factors are the primary reasons in RWPT technology behind the higher power transfer 
efficiency over long spaces. Additionally, it is described that RWPT and IWPT technology will unify in the future in spite of their existing differences.

\section{Basic Modelling of RWPT System}

The aim of this paper is to design the model of RWPT by varying the circuit parameters including capacitances, inductances and operating frequency. In order to find out the equation of efficiency, the analysis of the WPT model is carried out by using a typical two coils circuit model, which uses simple series-series compensation topology as shown in Fig. 6.

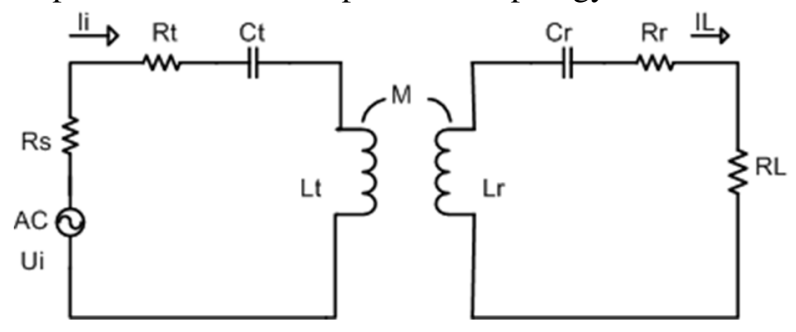

Fig. 6. Basic Circuit model of two coils RWPT system

By applying Kirchhoff's loop (or mesh) rule, Eq. 1 can be written as [4],

$$
\left[\begin{array}{cc}
Z_{t}+R s & j \omega L \\
j \omega L & Z_{r}+R_{L}
\end{array}\right]\left(\begin{array}{c}
I_{i} \\
I_{L}
\end{array}\right)=\left(\begin{array}{c}
U_{i} \\
0
\end{array}\right)
$$

Where $U_{i}$ is the source voltage of the transmitting coil at resonance frequency $(\omega)$, and $Z_{t}$ and $Z_{r}$ are the impedances of transmitting and receiving conductors, respectively. The mutual inductance $(M)$ between the transmitter coil and receiver coils is organized in terms of $\mathrm{k}$ and self-inductances of coils, which can be written as,

$$
M=k \sqrt{L_{t} L_{r}}
$$

And $\mathrm{Z}_{\mathrm{t}, \mathrm{r}}=\mathrm{R}_{\mathrm{t}, \mathrm{r}}+\mathrm{j} \omega L_{t, r}+1 / \mathrm{j} \omega C_{t, r}$

The efficiency $(\eta)$ is given by,

$$
\eta=\frac{\left|I_{L}\right|^{2} R_{L}}{\left|I_{i}\right|^{2} R_{t}+\left|I_{L}\right|^{2}\left(R_{L}+R_{r}\right)}
$$

The currents flowing through the transmitting coils and the receiving/load coil can be expressed by current divider rule.

$$
\begin{aligned}
I_{i} & =\frac{\left(R_{L}+Z_{r}\right) U_{i}}{\left(Z_{t}+R_{S}\right)\left(R_{L}+Z_{r}\right)+(\omega M)^{2}} \\
I_{L} & =\frac{(j \omega M) U_{i}}{\left(Z_{t}+R_{S}\right)\left(R_{L}+Z_{r}\right)+(\omega M)^{2}}
\end{aligned}
$$

From (3), it can be extracted that efficiency is associated with the parameters including input and output current, Load resistance $\mathrm{R}_{\mathrm{L}}$, source resistance $\left(R_{S}\right)$. According to (4) and (5), the input and output currents are related with mutual inductance $(M)$ resonance frequency $(\omega)$. Therefore, by optimizing the related circuit parameters efficiency can be improved. Furthermore, Impedance matching and adaptive shifting frequency method can be used to get maximum efficiency. Note that for getting maximum efficiency, the high frequency AC supply is required. However, it is quite difficult to implement high frequency (HF) AC 
supply. Therefore, it is of utmost importance to decrease the resonant frequency in order to have easy access to HF power electronics inverters. In this regard, the frequency decrease analysis is carried out by using circuit model as illustrated in Fig. 7, where the lumped capacitors for compensation are directly connected with supply.

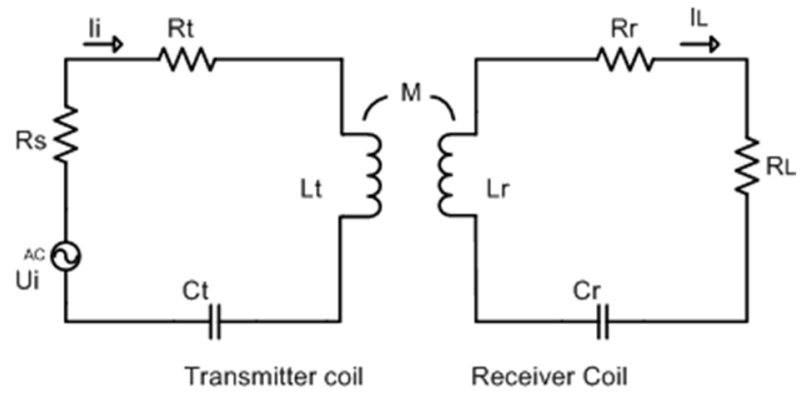

Fig. 7. Two-coil structure of RWPT system

From Fig. 7, the power transfer efficiency (PTE) in terms of mutual inductance and resonance frequency can be written as [24],

$$
\eta=\frac{\left(\frac{\omega M}{R}\right)^{2}}{1+\left(\frac{\omega M}{R}\right)^{2}+\frac{R_{L}}{R}} \frac{\frac{R_{L}}{R}}{1+\frac{R_{L}}{R}}
$$

For the simplification of the mathematical calculations, the supposition can be taken such as, $R_{t}=R_{r}=R$, then the load matching factor $(\mathrm{Lm})$ and the transfer quality factor $(Q)$ can be expressed as,

$$
\begin{aligned}
& Q=\frac{\omega M}{R} \\
& L m=\frac{R_{L}}{R}
\end{aligned}
$$

Now (8) can be simplified as,

$$
\eta=\frac{(Q)^{2}}{1+(Q)^{2}+L m} \frac{L m}{1+L m}
$$

From (9), it can be concluded that the transfer quality factor is directly related with the transfer efficiency. Hence, by increasing quality factor $(Q)$, the higher efficiency can be obtained, but it is essential to choose the optimized value of load-matching-factor $L m$, in order to obtain higher efficiency. It should be noted that typically a RWPT system requires high frequency in several $\mathrm{MHz}$ range to get high efficiency. But for examining the effects of decreasing the frequency, WPT system with reduced frequency of few $\mathrm{MHz}$ can be used. Note that, when the resonance condition arises, the coil reactive impedance reaches to zero, in that situation, the resonance frequency of the transmitting coil and receiving coil can be expressed as,

$$
\omega_{t, r}=\frac{1}{\sqrt{L_{t, r} C_{t, r}}}
$$

From (10), it can be easily understood that the resonance frequency can be reduced by enhancing the inductance or the capacitance. However, an optimized selection of inductance and capacitance is obligatory to obtain the higher efficiency. 


\subsection{Typical design of RWPT model}

Usually a system of wireless power transfer has need of High Frequency Alternating Current (AC) supply to generate strong electromagnetic field. Thereafter that electromagnetic field can be captured by any receiving antenna. Note that for capturing the maximum amount of electromagnetic field, the transmitting and receiving antennas must be resonating at an identical frequency. Typically, an Impedance matching technique is used for getting the identical resonating frequency. After the maximum capture of electromagnetic waves, the electromagnetic energy is converted back to the electrical energy and then supplied to load. Fig.8. Shows the typical block diagram of RWPT system.

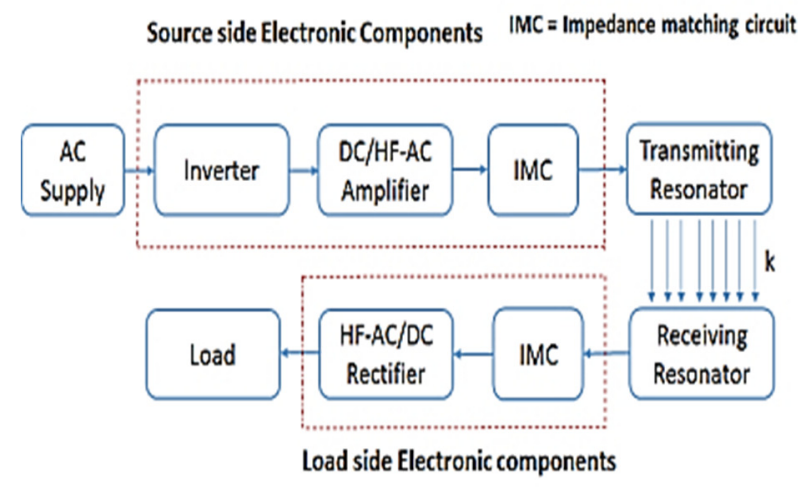

Fig. 8. Block diagram of the RWPT system

In this paper, the modeling and simulation of a resonant wireless power transfer is carried by using ADS designing software. The power amplitude of the model can vary from few Watts to tens of Watts and it can be used for transferring the power in range of tens of centimeters distances. The designed model uses series-parallel mixed circuit structure. Fig. 9 shows the schematic diagram of the model and its circuit parameters are given in Table 1. Note that for verification purposes, some parameter values are taken from [9] and the parameters are further tuned for better results.
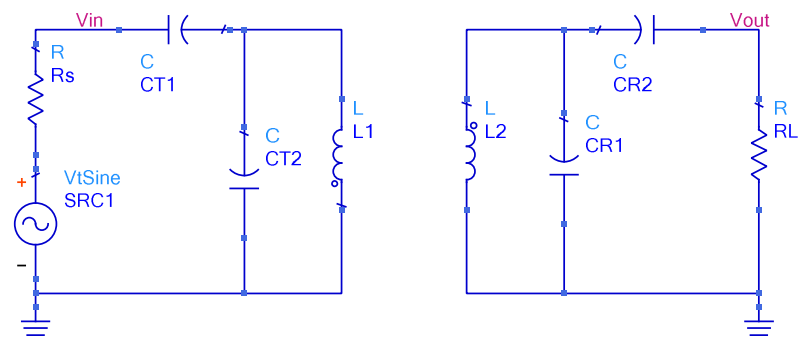

Fig. 9. Schematic of designed model using ADS

In order to find out the efficiency equation for series-parallel-mixed topology model an impedance seen from source side can be derived from Fig. 9. For finding impedances equations Fig 9. is redrawn as Fig. 10 (a) and $\mathrm{Z} 2$ is calculated as given in (11). For simplification, the capacitor names have been altered in Fig. 10 as follow; CT1=C1, CT2=C2, $\mathrm{CR} 1=\mathrm{C} 3$ and $\mathrm{CR} 2=\mathrm{C} 4$.

$$
Z_{2}=\frac{1+j \omega C_{4} R_{L}}{j \omega C_{3}+j \omega C_{4}-\omega^{2} C_{3} C_{4} R_{L}}
$$


Table. 1. Parameters used in designed RWPT model

\begin{tabular}{|l|l|}
\hline Parameter Name & Parameter Value \\
\hline Frequency & $4 \mathrm{MHz}$ \\
\hline Voltage Amplitude & $20 \mathrm{~V}$ and $40 \mathrm{~V}$ peak to peak \\
\hline $\mathrm{R}_{\mathrm{S}}$ & $50 \mathrm{ohms}$ \\
\hline $\mathrm{R}_{\mathrm{L}}$ & $100 \mathrm{ohms}$ \\
\hline $\mathrm{CT} 1$ & $52 \mathrm{pF}$ \\
\hline $\mathrm{CT} 2$ & $315 \mathrm{pF}$ \\
\hline $\mathrm{L} 1$ and L2 & $4.31 \mathrm{micro}$ Henry \\
\hline $\mathrm{CR} 1$ & $330 \mathrm{pF}$ \\
\hline $\mathrm{CR} 2$ & $37 \mathrm{pF}$ \\
\hline $\mathrm{k}$ (coupling factor) & 0.009 \\
\hline
\end{tabular}

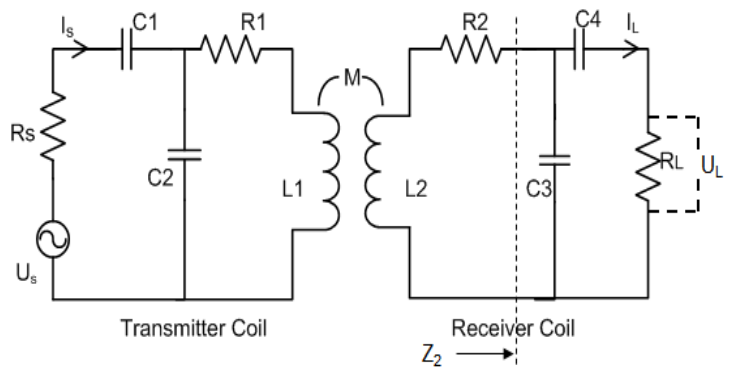

(a)

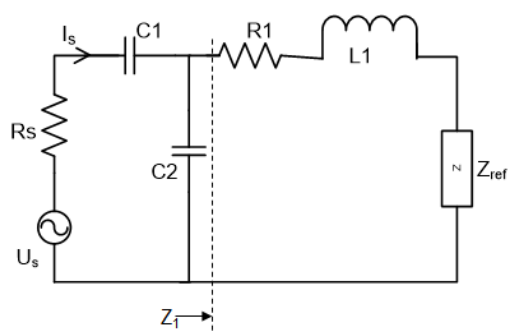

(b)

Fig. 10. Schematic of mixed topology for finding impedances

From Fig. 10 (b), Z reflected can be calculated, that is the reflected impedance from secondary side to primary side. Which is given by (12),

$$
Z_{\text {ref }}=\frac{(\omega M)^{2}}{j \omega L_{2}+R_{2}+Z_{2}}
$$

Moreover, Z1 can be calculated by (13), which comprises of both inductances L1 and L2 and mutual inductance $M$. For finding the efficiency we need total impedance $\left(Z_{\text {in }}\right)$ from primary side which can be seen by voltage source $U_{S}$ and that is given by (14).

$$
\begin{aligned}
& Z_{1}=R_{1}+\mathrm{j} \omega L_{1}+\frac{(\omega M)^{2}}{j \omega L_{2}+R_{2}+Z_{2}} \\
& Z_{\text {in }}=R_{S}+\frac{1}{j \omega C_{1}+\omega C_{2}}+R_{1}+j \omega L_{1}+\frac{(\omega M)^{2}}{j \omega L_{2}+R_{2}+Z_{2}}
\end{aligned}
$$

The formula for efficiency of series-parallel-mixed topology can be described by following equation[34].

$$
\eta_{\text {mixed }}=\frac{|A \cdot B \cdot C|^{2}\left|Z_{i n}\right|^{2}}{\left(R_{L} \cdot \operatorname{Real}\left[Z_{i n}\right]\right)}
$$

In (15) $\mathrm{A}, \mathrm{B}$ and $\mathrm{C}$ are described as follows[9]. The complex value of $\mathrm{Z}_{\text {in }}$ is given in (14). Note that the Real[ $\left.Z_{\text {in }}\right]$ is the real part of complex $Z_{\text {in }}$. 


$$
\begin{gathered}
A=\frac{C_{1}}{C_{1}+C_{3}+j \omega C_{1} C_{3} R_{S}} \\
B=\frac{j \omega C_{4} R_{L}}{1+j \omega C_{4} R_{L}+\left(j \omega C_{3}+j \omega C_{4}-\omega^{2} C_{3} C_{4} R_{L}\right) R_{2}} \\
C=\frac{\left(j \omega K \sqrt{L_{1} L_{2}}\left(R_{2}+\frac{1+j \omega C_{4} R_{L}}{j \omega C_{3}+j \omega C_{4}-\omega^{2} C_{3} C_{4} R_{L}}\right)\right.}{\left(j \omega L_{1}+R_{1}+\frac{1+j \omega C_{1} R_{S}}{j \omega C_{1}+j \omega C_{3}-\omega^{2} C_{1} C_{2} R_{S}}\right) \cdot\left(j \omega L_{2}+R_{2}+\frac{1+j \omega C_{2} R_{L}}{j \omega C_{3}+j \omega C_{4}-\omega^{2} C_{2} C_{4} R_{L}}\right)+(\omega K)^{2} L_{1} L_{2}}
\end{gathered}
$$

From the above analysis of mixed topology model, the efficiency of the model can be calculated theoretically by equation (15).

\section{Simulations of the designed model}

In this section, the simulation results of the RWPT model are provided and discussed. The optimization of the model is carried out by varying the model parameters, which consist of resistances, inductances and capacitances. The schematic of the model as shown in Fig. 9 is simulated for two different input voltage amplitudes in order to check the feasibility of the model for several power levels. Initially, $20 \mathrm{~V}$ peak-to-peak voltage and thereafter $40 \mathrm{~V}$ peakto-peak voltage is applied. The obtained results illustrate that the output waveform is in almost similar shape as input waveform shape. This behavior of waveform shape shows that the WPT model has minimum distortions and disturbances during the power transfer from source coil to load coil. Fig. 11 and Fig. 12 show the input and output waveforms of current and voltages with applied voltage of $20 \mathrm{~V}$ peak-to-peak value. The achieved efficiency with voltage amplitude of $20 \mathrm{~V}$ is around $49.10 \%$. Note that the efficiency is computed by dividing output power to input power. In simulation results, for the simplification purposes, the output and input powers are calculated by multiplying peak values of corresponding voltage and currents.

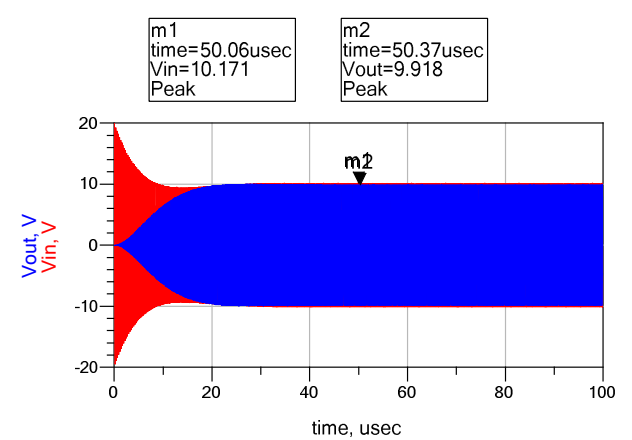

(a)

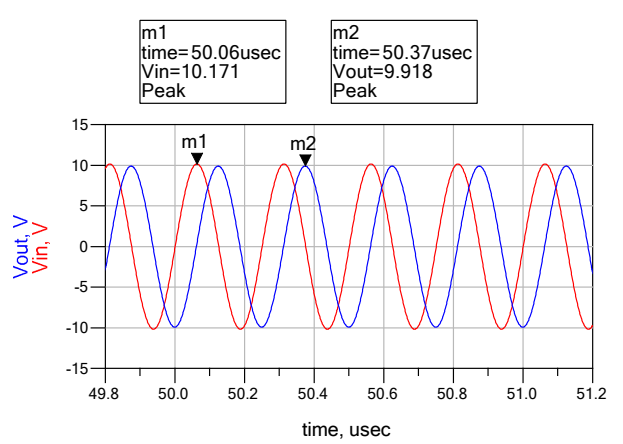

(b)

Fig. 11. Input and output voltage waveforms of the designed model for supply voltage of $20 \mathrm{~V}$ (peak to peak). (a) Full view (b) Zoom in view 


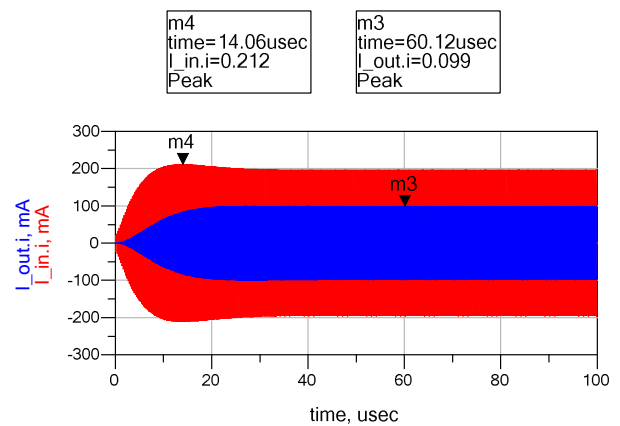

(a)

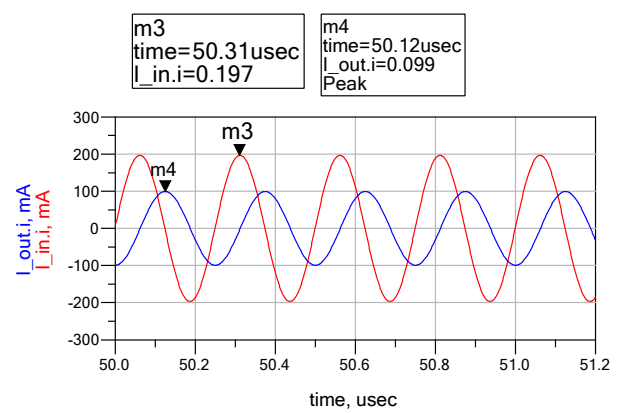

(b)

Fig. 12. Input and output current waveforms of the designed model for supply voltage of $20 \mathrm{~V}$ (peak to peak). (a) Full view (b) Zoom in view

The input and output waveforms of voltage and current using $40 \mathrm{~V}$ amplitude are given in Fig. 13 and Fig. 14. The related efficiency is $49.52 \%$ approximately.

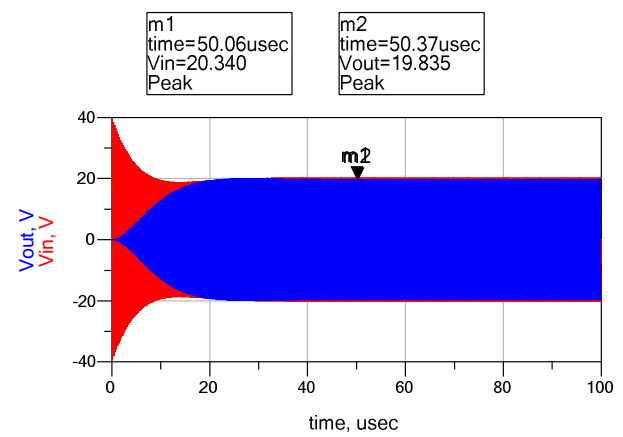

(a)

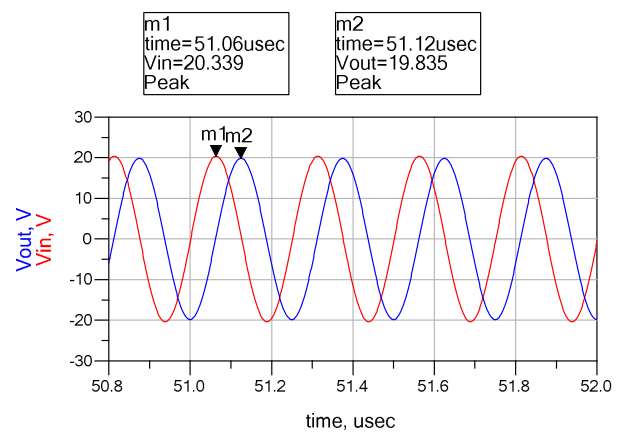

(b)

Fig. 13. Input and output voltage waveforms of the designed model for supply voltage of $40 \mathrm{~V}$ (peak to peak). (a) Full view (b) Zoom in view

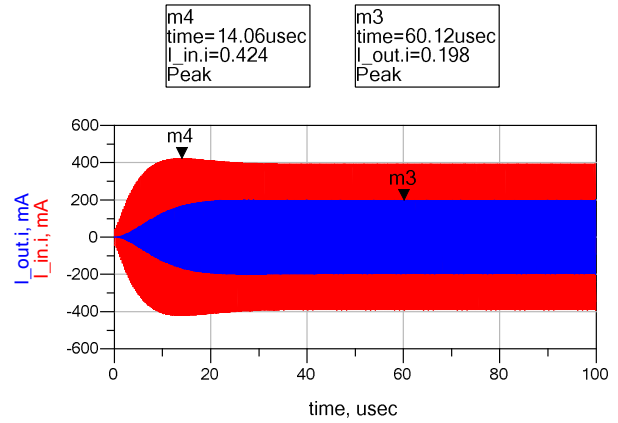

(a)

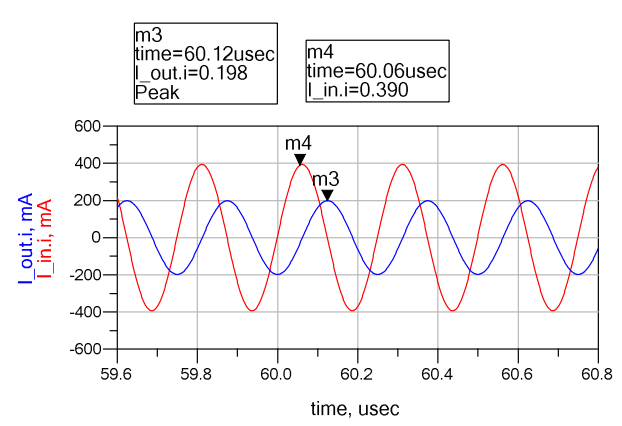

(b)

Fig. 14. Input and output current waveforms of the designed model for supply voltage of $40 \mathrm{~V}$ (peak to peak). (a) Full view (b) Zoom in view

The results of the efficiency calculation show that at different voltage levels, there is very slighter change in efficiency. From the simulation results, it can be concluded that this model is appropriate for different power levels and can be further optimized for better results. Note that the coupling factor $(k)$, which defines the distance between coils, has been kept small and the designed model is suitable for transferring the power with good efficiency in 
centimeters range. The full view of the simulation results indicates that at the startup of the simulation, there are some disturbances in waveform, but within 10-20 microseconds, the system starts stabilizing the waveform and afterwards the waveform can be seen in stable condition without any disturbances. Furthermore, it is worth mentioning that value of $\mathrm{R}_{\mathrm{L}}$ used in this model is not same as $\mathrm{R}_{\mathrm{S}}$, it indicates that the mixed resonant model can be a good choice even for unsymmetrical loads.

\section{Conclusion}

The design and analysis of resonant wireless power transfer system are conducted using circuit theory and simulations are performed using ADS design software. The circuit equations for series-series and series-parallel-mixed topology model are investigated for calculating the efficiency. Two different input voltage levels have been used to test the performance of the series-parallel-mixed topology model. At $20 \mathrm{~V}$ peak-to-peak voltage, the obtained efficiency is around $49.10 \%$. When the input voltage is increased to $40 \mathrm{~V}$ peak-topeak value, then the slight higher efficiency around $49.52 \%$ is achieved. In addition, the efficiency analysis of the model is discussed in the paper and the effects of the resonance frequency on the functioning of the WPT system are also presented.

The Authors would like to thank Universiti Teknologi PETRONAS for providing the research fund for this study.

\section{References}

1. $\quad$ N. Tesla, "Apparatus for transmitting electrical energy," (1914).

2. S. Y. R. Hui, W. Zhong, and C. K. Lee, IEEE Trans. Power Electron. 29(9), pp. 4500-4511 (2014).

3. B. L. Cannon, J. F. Hoburg, D. D. Stancil, and S. C. Goldstein, Power Electron. IEEE Trans. 24(7), pp. 1819-1825 (2009).

4. D. M. Vilathgamuwa and J. P. K. Sampath, Wireless Power Transfer (WPT) for Electric Vehicles (EVs)_Present and Future Trends, in Plug In Electric Vehicles in Smart Grids pp. 33-60, (Springer, 2015).

5. S. Das Barman, A. W. Reza, N. Kumar, M. E. Karim, and A. B. Munir, Renew. Sustain. Energy Rev. 51, pp. 1525-1552 (2015).

6. D. Kürschner, C. Rathge, and U. Jumar, Ind. Electron. IEEE Trans. 60(1), pp. 372381 (2013).

7. S. L. Ho, J. Wang, W. N. Fu, and M. Sun, Magn. IEEE Trans. 47(5), pp. 15221525 (2011).

8. A. P. Sample, D. Meyer, and J. R. Smith, Ind. Electron. IEEE Trans. 58(2), pp. 544-554 (2011).

9. L. Chen, S. Liu, Y. C. Zhou, and T. J. Cui, Ind. Electron. IEEE Trans. 60(1), pp. 339-349 (2013).

10. T. P. Duong and J.-W. Lee, Energies 8(8), pp. 7593-7617 (2015).

11. " "http://www.tfcbooks.com/articles/witricity.htm," .

12. " "http://www.teslasociety.com/teslashorelab.htm," .

13. A. Karalis, J. D. Joannopoulos, and M. Soljačić, Ann. Phys. (N. Y). 323(1), pp. 3448 (2008).

14. A. Kurs, A. Karalis, R. Moffatt, J. D. Joannopoulos, P. Fisher, and M. Soljačić, Science (80-. ). 317(5834), pp. 83-86 (2007).

15. B. Zhu, J. Li, W. Hu, and X. Gao, Int. J. U-\& E-Service, Sci. Technol. 8(3), (2015).

16. C. E. Greene, D. W. Harrist, and M. T. McElhinny, "Powering cell phones and similar devices using RF energy harvesting," (2007). 
17. J. Sallán, J. L. Villa, A. Llombart, and J. F. Sanz, Ind. Electron. IEEE Trans. 56(6), pp. 2140-2149 (2009).

18. "https://www.wirelesspowerconsortium.com/tradeshows/20160616_taipei/," .

19. W. Junhua, S. L. Ho, W. N. Fu, and S. Mingui, Magn. IEEE Trans. 47(10), pp. 2616-2619 (2011).

20. K. Fotopoulou and B. W. Flynn, Magn. IEEE Trans. 47(2), pp. 416-430 (2011).

21. S. Krishnan, S. Bhuyan, V. P. Kumar, W. Wenjiang, J. A. Afif, and L. Khoon Seong, Frequency Agile Resonance-Based Wireless Charging System for Electric Vehicles, in Electr. Veh. Conf. (IEVC), 2012 IEEE Int. pp. 1-4 (2012).

22. A. Kurs, R. Moffatt, and M. Soljačić, Appl. Phys. Lett. 96(4), pp. 44102 (2010).

23. C. Sanghoon, K. Yong-Hae, S.-Y. Kang, L. Myung Lae, L. Jong-Moo, and T. Zyung, Ind. Electron. IEEE Trans. 58(7), pp. 2906-2914 (2011).

24. Z. Yiming, Z. Zhengming, and C. Kainan, Power Electron. IEEE Trans. 29(3), pp. 1058-1063 (2014).

25. J. I. Agbinya and N. F. A. Mohamed, Int. J. Electr. Power Energy Syst. 63, pp. 1047-1056 (2014).

26. R. Huang, B. Zhang, D. Qiu, and Y. Zhang, Magn. IEEE Trans. 50(11), pp. 1-4 (2014).

27. D. Ahn and S. Hong, Ind. Electron. IEEE Trans. 61(3), pp. 1193-1203 (2014).

28. P. Changbyung, L. Sungwoo, C. Gyu-Hyeong, and C. T. Rim, Power Electron. IEEE Trans. 30(2), pp. 817-827 (2015).

29. T. Imura and Y. Hori, Ind. Electron. IEEE Trans. 58(10), pp. 4746-4752 (2011).

30. W. Zhang, S.-C. Wong, C. K. Tse, and Q. Chen, Power Electron. IEEE Trans. 29(6), pp. 2979-2990 (2014).

31. S. Li and C. C. Mi, Emerg. Sel. Top. Power Electron. IEEE J. 3(1), pp. 4-17 (2015).

32. C. A. Tucker, K. Warwick, and W. Holderbaum, Int. J. Electr. Power Energy Syst. 47, pp. 235-242 (2013).

33. X. Wei, Z. Wang, and H. Dai, Energies 7(7), pp. 4316-4341 (2014).

34. S. Wang, J. Chen, Z. Hu, and M. Liu, Study on Series-Parallel Mixed-Resonance Model of Wireless Power Transfer via Magnetic Resonance Coupling, in Prog. Electromagn. Res. Symp. pp. 2941-2945 (IEEE, 2016 